\title{
Some peculiarities of aluminum and titanium alloys stamping
}

\author{
Evgeny Galkin ${ }^{1, *}$ \\ ${ }^{1}$ Moscow Aviation Institute, Moscow, Russia
}

\begin{abstract}
The research is devoted to the technological peculiarities of hemispherical shells of high duty-alloys. The article shows the results of finite-element modelling process and the methodology of technological junction during punching.
\end{abstract}

High-duty alloys are applied for production of essential components, also of hemispherical shells, which can be used as bottom of high pressure holding capacities.

Titanium alloy is the main material for their production due to its high endurance and specific rigidity. Due to alloy's peculiarity it is consistent to apply stamping through stretching by a moldable plumb die into a stiff matrix. Joint plastic deformation of plumb and blank part material affords to form the piece by the effect of blank part pressing by the moldable die into the matrix fuller [1,2].

Due to the moldable deformation of the die on its border with the blank part, it creates the conditions of active friction which have a positive impact on the changing the form of the blank part and the possibility of its natural thinning. The process scheme is presented on Fig. 1 .

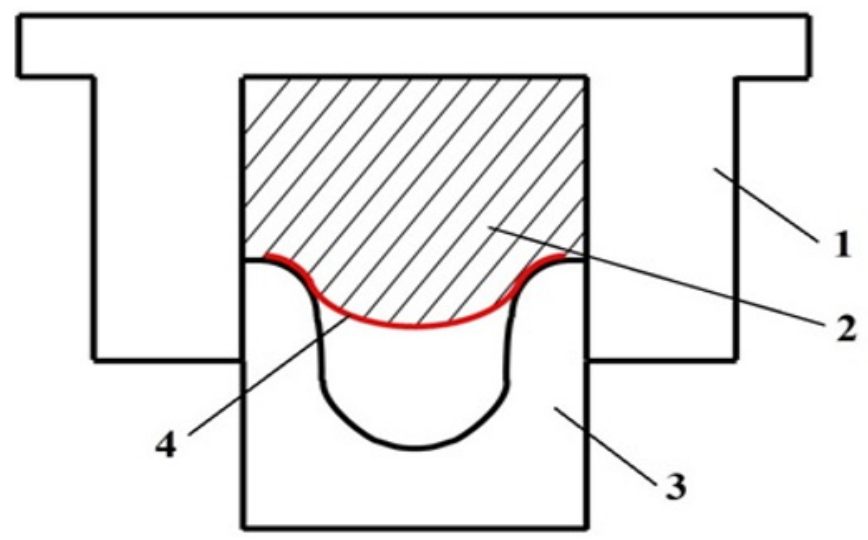

Fig. 1. Geometric scheme of process, where 1 is container, 2 - plumb die, 3 - matrix, 4 - blank part

* Corresponding author: e.v.galkin@yandex.ru 
A research aimed at investigation of strain-stress state of the blank part sheet in the process of stamping-stretching showed that the development of the technological process possesses some peculiarities. The blank part is produced of titanium alloy ST-A90. The mathematical modelling of the process was accomplished by the finite-element method with the usage of software product DEFORM - 2D, oriented at solving problems of metal deformation process.

Technical valuation of stretching of details supposes the definition of accessible deformation degree, identifying of the needed number of consequent steps of stretching and calculation of step-by-step size of details.

The accessible deformation degree is calculated according to experimentally found and practically checked elongation ratio K. A physical experiment was carried out before the modelling to define the moment of blank part fracturing at its stretching at the first junction. During the experiment it was specified that the admissible limit value for elongation ratio $\mathrm{K}$ was 0,9 . Also the gauge of wall of the blank part surface was measured (Picture 2, Table 1).

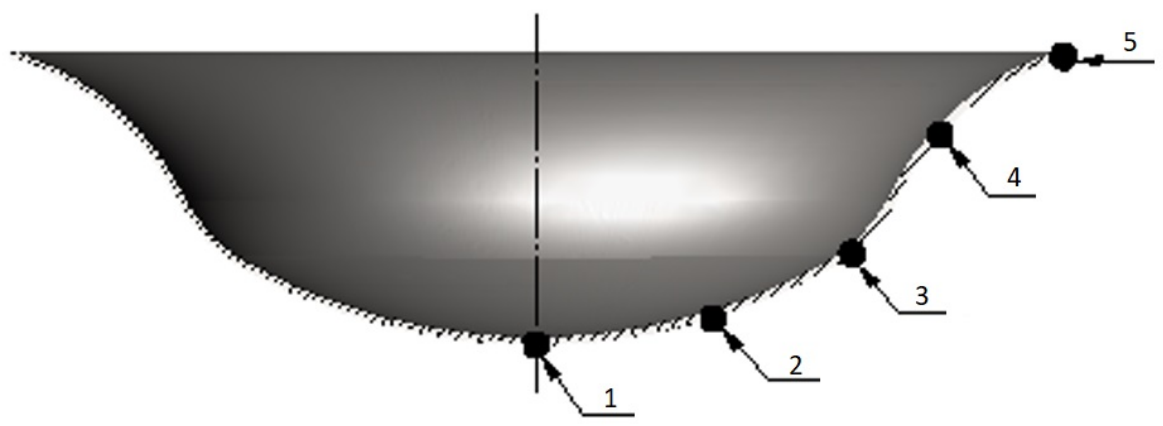

Fig. 2. Points (1-5), in which the gauge was measured

Table 1. Results of gauge measurements

\begin{tabular}{|c|c|}
\hline Point & Gauge of wall after punching, mm \\
\hline 1 & 2,92 \\
\hline 2 & 3,05 \\
\hline 3 & 3,02 \\
\hline 4 & 3,02 \\
\hline 5 & 3,02 \\
\hline
\end{tabular}

Comparative analysis of experimental data was carried out to define the quantitative outcome of the stress-strain state of the blank part and with the help of mathematical model which had the following initial datum: geometric size of the die, blank part, matrix and container: temperature equals to $20^{\circ} \mathrm{C}$; characteristics of the blank part material (ST-A90) and the die; blank part wall gauge equals to $3 \mathrm{~mm}$, speed of matrix slide is $1 \mathrm{~mm} / \mathrm{sec}$. The mathematical model was developed on the basis of these data (Figure 3). 


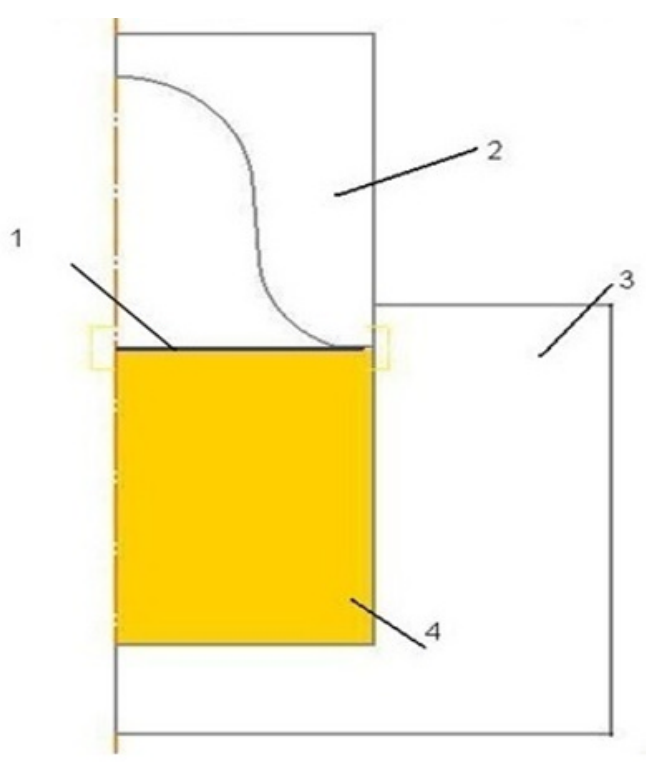

Fig. 3. Mathematical model of process, where: 1-blank part, 2 - matrix, 3 - container, 4 - plumb die

The conducted calculation showed a high convergence of experimental and maths modelling results, the deviation was not higher than 5\% (Table 2).

Table 2. Comparison of math modeling and experimental results

\begin{tabular}{|l|l|l|l|}
\hline \multirow{2}{*}{ Point } & \multicolumn{2}{|l|}{ Blank part gauge, mm } & \multirow{2}{*}{ Deviation, \% } \\
\cline { 2 - 3 } & Real process & Modelling & \\
\hline 1 & 2,92 & 2,84 & 2,7 \\
\hline 2 & 3,05 & 2,9 & 4,9 \\
\hline 3 & 3,02 & 3,03 & 0,3 \\
\hline 4 & 3,02 & 3,03 & 0,3 \\
\hline 5 & 3,02 & 3,17 & 5,0 \\
\hline
\end{tabular}

The calculation results showed that the maximum degree of deformation and strain intensity are achieved in the border zone between matrix and die. There we can see the maximum degree of compressing tang and normal stress and moderate values of stretching stress. The presence of two compressing components of stress tensor shows the favorableness of stress state.

The analysis of the allocation of the strain-stress state of the blank part shows that the intensity of strain almost reaches the maximum permissible for alloy ST-A90 (600-750 MPag). Nevertheless, taking into account the favorableness of stress state scheme for metal punching, these values should be considered as the maximum possible ones. This fact allowed to confirm that the developed mathematical model is appropriate and the quantitative results are absolutely adequate to the real situation. It also allows to investigate effectively 
the strain-stress state of the material, as well as the processes of wall thinning and surface elongation.

Another perspective material which can be used for hemispherical shells production is sliced aluminum PAS which consists of Al-Zn-Mg alloys and AA1135. PAS possesses a better moldability, low weight characteristics as well as being almost as hard as titanium.

The above mentioned methodology for definition of permissible stretching value for the blank part made of aluminum (PAS), showed the threshold limit stretching value of $\mathrm{K}$ which was equal to 0,75 . The gauge of wall on its surface was also measured. On the basis of the stretching value, we could calculate the quantity of stamping junctions for the production of hemispherical shells.

The above mentioned methodology allowed to calculate the value of strain-stress state of the blank part, the degree of wall thinning and elongation of its surface. Table 3 shows the values of the wall gauge in the real process and in the process of modelling. The deviation of the calculation was not higher than $7 \%$.

Table 3. Values of the wall gauge in the real process and in the process of modelling

\begin{tabular}{|c|c|c|c|}
\hline \multirow{2}{*}{ Point } & \multicolumn{2}{|c|}{ Blank part gauge, mm } & \multirow{2}{*}{ Deviation, \% } \\
\cline { 2 - 3 } & Real process & Modelling & \\
\hline 1 & 2,93 & 2,82 & 3,7 \\
\hline 2 & 3,03 & 2,91 & 3,96 \\
\hline 3 & 3,01 & 2,91 & 3,3 \\
\hline 4 & 3,04 & 3,15 & 3,6 \\
\hline 5 & 3,04 & 3,24 & 6,57 \\
\hline
\end{tabular}

\section{Conclusion}

1. The stretching of thin walled hemispherical shells of aluminum and titanium alloys with the application of moldable die is a perspective method of production which allows to receive an item with a minimum thinning and favorable conditions of the strain-stress state.

2. The experiment allowed to develop a methodology of projecting which can be used for stretching of hemispherical shells made of different types of materials.

\section{References}

1. V.I. Galkin, Ye.D. Veingerova Investigation of strain-stress state of a steel blank part AISI 304 during stretching of scale-sale bottoms in instrumental dies. Engineering technology, 8, (2006)

2. A.P. Petrov, V.I. Galkin, A.R. Paltievich Application of the finite-element analysis by development of technological processes of metals pressure deforming. Scientific investigation Vestnik MATI, 12 (84), (2007), pp. 57-64 